\title{
Klotho inhibits human follicular thyroid cancer cell growth and promotes apoptosis through regulation of the expression of stanniocalcin-1
}

\author{
DONG DAI*, QI WANG* ${ }^{*}$ XIAOFENG LI, JIANJING LIU, \\ XIAOYING MA and WENGUI XU
}

\begin{abstract}
Department of Molecular Imaging and Nuclear Medicine, Tianjin Medical University Cancer Institute and Hospital, National Clinical Research Center for Cancer, Key Laboratory of Cancer Prevention and Therapy, Tianjin 300060, P.R. China
\end{abstract}

Received June 17, 2015; Accepted July 29, 2015

DOI: $10.3892 /$ or.2015.4358

\begin{abstract}
The new anti-aging gene Klotho has been identified as a multi-functional humoral factor which influences multiple biological processes, including tumor progression. Although ample evidence indicates that Klotho plays important roles in cervical, lung and breast cancer, the role and mechanism of Klotho in thyroid cancer are still unclear. The present study aimed to investigate the effects and mechanisms of Klotho in human thyroid cancer cell lines FTC133 and FTC238. Klotho overexpression markedly reduced thyroid cancer FTC133 and FTC238 cell proliferation and enhanced apoptosis, whereas, Klotho silencing in the FTC133 and FTC238 cells increased cell growth. Moreover, soluble human KL1 (sKL) and Klotho overexpression had a similar effect on FTC133 and FTC238 cell growth. A high level of Klotho was also found to be associated with a low level of stanniocalcin 1 (STC1) in both the FTC133 and FTC238 cell lines. STC1 silencing significantly inhibited thyroid cancer cell proliferation, whereas recombinant human STC1 (hSTC1) markedly enhanced cell proliferation. In addition, our study demonstrated that hSTC1 treatment attenuated Klotho-induced inhibition of cell proliferation and promotion of apoptosis. Our data revealed the existence of a moderating effect between Klotho and STC1, where Klotho may inhibit thyroid tumor progression by inhibiting the tumor marker level of STC1.
\end{abstract}

\section{Introduction}

Thyroid cancer is the most common endocrine malignancy, and the number of new cases diagnosed worldwide has

Correspondence to: Dr Wengui Xu, Department of Molecular Imaging and Nuclear Medicine, Tianjin Medical University Cancer Institute and Hospital, Huan-Hu-Xi Road, Ti-Yuan-Bei, He Xi District, Tianjin 300060, P.R. China

E-mail: xu_wengui@163.com

${ }^{*}$ Contributed equally

Key words: stanniocalcin 1, Klotho, thyroid cancer, cell growth, apoptosis increased in the last decade (1). Thyroid cancer is classified into undifferentiated and differentiated cancer, and the vast majority of thyroid cancers $(\sim 80 \%)$ are differentiated thyroid cancers (DTCs) (2). DTCs consist of two cellular types: papillary thyroid cancers (PTCs) and differentiated follicular thyroid cancers (FTCs). Various effective treatments have been used for thyroid cancer therapy, such as complete thyroidectomy followed by radioiodine therapy (3). These patients have a low mortality rate and excellent prognosis. However, a subset of patients that develops distant metastases presents with a high disease recurrence rate and poor prognosis and the disease may develop into anaplastic thyroid cancer with a fatal outcome $(4,5)$. Therefore, research on the molecular mechanisms involved in thyroid carcinogenesis will facilitate the development of more effective therapy for this cancer.

Ample evidence indicates that aging is the main risk factor for cancer, and most cancers are diagnosed in individuals over 55 years of age (6). Currently, Klotho has recently been identified as a new anti-aging gene and has gained much attention (7). Klotho (KL), a 1012-amino acid type I single-pass transmembrane protein, is widely expressed in various types of tissues, such as brain, kidney, various exocrine and endocrine tissues, including the thyroid gland (8-10). The Klotho gene is composed of 5 exons $(8,11)$ and contains an intracellular domain and an extracellular domain. The extracellular domain of Klotho is composed of two domains, KL1 and KL2, with weak homology (10), while the intracellular domain is short ( 10amino acid long) which has no known functional domain (6). The Klotho gene plays roles in various diseases in mice, such as arteriosclerosis, osteoporosis, endothelial dysfunction, Parkinsonian gait and cognitive impairment (12-14). In humans, Klotho is involved in reduced human longevity and coronary artery disease (15). Recently, Klotho was identified as a potential tumor suppressor, and ample evidence suggests that Klotho expression influences human breast, hepatocarcinogenesis, cervical and lung cancers (7,16-18). However, the functional role and specific molecular mechanism of Klotho in thyroid cancer remain unclear.

Ample laboratory data indicate that the Klotho gene could regulate the expression of STC1 and STC2 in the kidney (19). Stanniocalcin (STC) is a secreted glycoprotein hormone and 
has two isotypes, STC1 and STC2. Human STC1 is encoded by a single copy gene which is localized at chromosome 8p11.2-p21 (20). STC1 was originally discovered in mammals and in the endocrine glands of the kidneys, and stannius corpuscles of bony fishes (21). STC1 is expressed in a wide variety of tissues, such as the heart, prostate, adrenal gland, lung, kidney, liver and thyroid gland (22-24). Multiple lines of evidence show that mammalian STC1 was cloned as a cancer-related gene (25). Growing evidence suggests that STC1 expression may play an important role in human cancers, and high STC1 gene expression has been found in colorectal, breast, hepatocellular, carcinomas and thyroid cancers (26-29). In the present study, we investigated whether Klotho expression is associated with the development of thyroid cancer via regulation of STC1 protein expression. We found that Klotho overexpression significantly inhibited human thyroid cancer cell growth and promoted apoptosis, and suppressed the expression of STC1.

\section{Materials and methods}

Cell culture and transfection. Human thyroid cancer cell lines established from primary thyroid cancer (FTC133) and distant metastases (FTC238) (both from ECACC, Wiltshire, UK) were used in this study. Cells were cultured in RPMI1640 medium (Invitrogen, Carlsbad, CA, USA) supplemented with $5 \%$ FCS, $1 \%$ glutamine and $1 \%$ penicillin-streptomycin. Klotho-overexpressing cells were constructed with the human Klotho expression plasmid (pCDNA3.1-Klotho) using Lipofectamine 2000 (Invitrogen) following the manufacturer's instructions (30).

Cell viability assay. Human thyroid cancer cells in exponential growth $\left(5 \times 10^{4}\right.$ cells/well $)$ were seeded into a 96 -well sterile culture plate. Cells were transfected with the Klotho vector or the control vector for $72 \mathrm{~h}$. Thyroid cancer cell viability was determined using the CellTiter-Glo Luminescent Cell Viability Assay kit following the manufacturer's instructions (Promega, Madison, WI, USA).

MTT assay. Human thyroid cancer cell proliferation was measured by the 3-(4,5-dimethylthiazol-2-yl)-2,5-diphenyltetrazolium bromide (MTT) assay according to a previously described method (6). The thyroid cancer cells $\left(5 \times 10^{4}\right.$ cells/ well) were plated in a 96-well culture plate for $24 \mathrm{~h}$. The cells were then transfected with Klotho and cultured for $72 \mathrm{~h}$, and $20 \mu \mathrm{l}$ MTT $(10 \mathrm{mg} / \mathrm{ml})$ was then added. The reaction was terminated after an additional $4 \mathrm{~h}$ of incubation. DMSO (200 $\mu \mathrm{l}$ ) was added to the cells for $10 \mathrm{~min}$. The optical density (OD) value of each well was measured at $570 \mathrm{~nm}$.

Nucleosome ELISA assay for detection of apoptosis. Human thyroid cancer cell apoptosis was carried out using the Nucleosome ELISA kit as previously described (31). FTC133 and FTC238 cells were harvested. Nucleosome ELISAs were performed according to the manufacturer's instructions (Oncogene Research Products, Cambridge, MA, USA).

Caspase-3 activity assay. Caspase-3 activity was detected using the Caspase-Glo ${ }^{\circledR} 3 / 7$ assay (Promega) according to the manufacturer's instructions.
Quantitative RT-PCR. Quantitative RT-PCR was performed as previously described (22). Total RNA was isolated from human thyroid cancer cells and reverse transcribed into cDNA using SuperScript ${ }^{\mathrm{TM}}$ III Reverse Transcriptase (Invitrogen). PCR amplification was carried out by an ABI PRISM 7900 thermocycler using SYBR Premix Taq (Applied Biosystems). The following primer pairs were used for PCR amplification: STC1_F, human STC1-specific primers were forward, 5'-ATC ACATTCCAGCAGGCTTC-3' and reverse, 5'-CCTGAAGCC ATCACTGAGGT-3'; human Klotho-specific primers were forward, 5'-ACTCCCCCAGTCAGGTGGCGGTA-3' and reverse, 5'-TGGGCCCGGGAAACCATTGCTGTC- 3'. The reaction conditions was as follows: $95^{\circ} \mathrm{C}$ for $5 \mathrm{~min}$; followed by 40 cycles at $95^{\circ} \mathrm{C}$ for $15 \mathrm{sec} ; 60^{\circ} \mathrm{C}$ for $45 \mathrm{sec}$. Relative levels of gene expression are expressed relative to $\beta$-actin and calculated using the $2^{-\Delta \Delta \mathrm{CT}}$ method (32).

Western blot analysis. Proteins were collected from the cells transfected with the Klotho vector or the control vector. Cells were washed in PBS and lysed using RIPA lysis buffer. The concentration of protein was measured using the BCA kit. Samples $(40 \mu \mathrm{g})$ were subjected to SDS-PAGE and transferred to Immobilon-P Transfer Membranes (Millipore). Western blot anlaysis was conducted using the following primary and secondary antibodies: rabbit anti-Klotho polyclonal antibody, rabbit anti-STC1 polyclonal antibody, mouse anti- $\beta$-actin monoclonal antibody, HRP-conjugated rabbit anti-mouse IgG and HRP-conjugated goat anti-rabbit IgG. Bands were visualized using an electrochemiluminescence kit (Immulite Pyrilinks-D; Diagnostic Products Corporation, Los Angeles, CA, USA). Antibodies used in this study were all obtained from Abcam (Cambridge, MA, USA).

siRNA. Klotho and STC1 siRNA oligoribonucleotides and control siRNAs were obtained from Shanghai Sangon Co., Ltd. (Shanghai, China). Their sequences were as follows: Klotho siRNA oligoribonucleotides directed against 1274-1298, 5'-ACCA AGAGAGAUGAUGCCAAAUAU-3' and control siRNAs scrambled, 5'-CACGAGAUAGAGUAG AACCAACUAU-3'; STC1 siRNA oligonucleotides against 369-392 (33), 5'-GGTGCAGGAAGAGTGCTACAGCAAG TACGTAGGTTGCTGTAGCACTCTTCCTGCACCCTTT TTG-3'. The DNA oligonucleotides used to generate scrambled siRNA were 5'-GGCGCGCTTTGTAGGATTCGAT ACGTAAACGAATCCTACAAAGCGCGCTTTTTG-3'. The cells were transfected with Klotho or STC1 siRNA or scrambled using Lipofectamine 2000 (Invitrogen) following the manufacturer's instructions (9).

Statistical analysis. Results from at least three independent experiments are presented as the mean \pm SD. Statistical comparisons were performed using one-way ANOVA and Dunnett's test. All analyses were performed using SPSS 13.0 software (SPSS, Chicago, IL, USA).

\section{Results}

Overexpression of Klotho inhibits thyroid cancer cell survival and proliferation. Cell proliferation plays a crucial role in tumor progression and can regulate the fate of tumors 
A
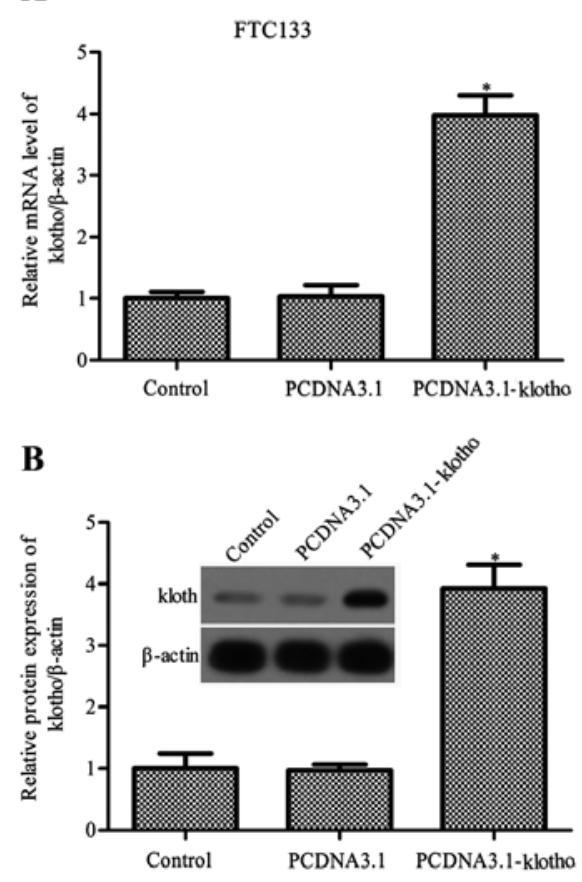

C

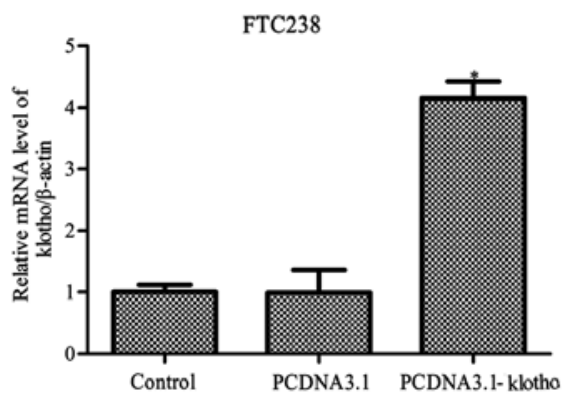

D

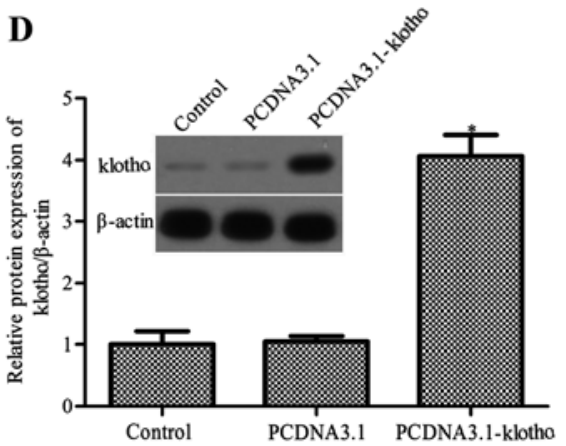

$\mathbf{E}$

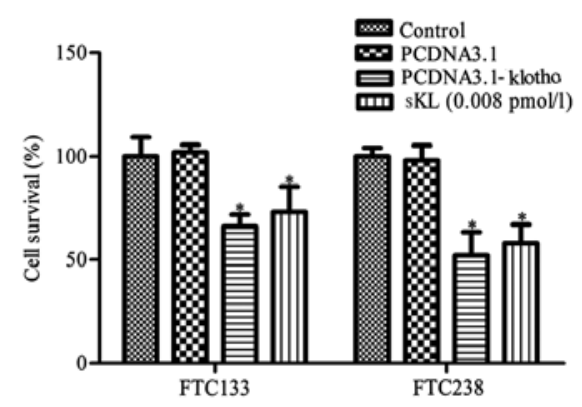

$\mathbf{F}$

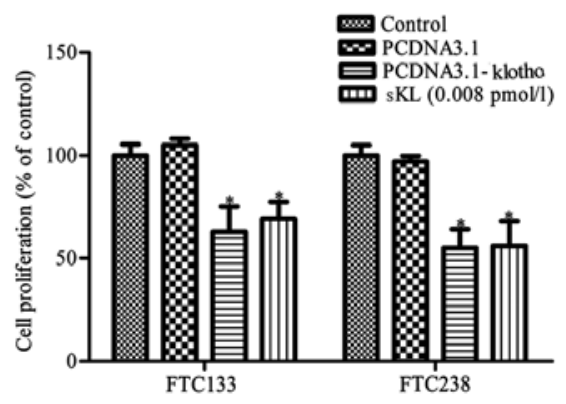

Figure 1. Effects of klotho overexpression on thyroid cancer cell survival and proliferation. (A-D) Klotho expression was assessed by RT-PCR and western blot analysis, respectively. FTC133 (A and B) and FTC238 (C and D) cells were transfected with either the pCDNA3.1-Klotho expression vector or control vector pCDNA3.1. (E) Cell survival was measured by MTT assay. (F) CCK-8 assay was used to detect the proliferation of FTC133 and FTC238 cells. Cells were transfected with pCDNA3.1-Klotho, control vector pCDNA3.1 or treated with 0.008 pmol/1 sKL. Experiments were performed in triplicate, and data shown are the mean results $\pm \mathrm{SD} ;{ }^{*} \mathrm{P}<0.05$ compared with the control group.

at any given time (30). Thus, in order to determine whether Klotho gene and protein expression is associated with thyroid cancer cell survival and proliferation, we constructed Klothooverexpressing cells using pCDNA3.1-Klotho plasmids. As shown in Fig. 1A-D, the mRNA and protein expression levels of Klotho in the FTC133 and FTC238 cells were markedly increased after pCDNA3.1-Klotho transfection. FTC133 and FTC238 cells were transfected with pCDNA3.1-Klotho or control pCDNA3.1, and then the cell viability was assessed by MTT assay. Klotho expression significantly inhibited FTC133 and FTC238 cell survival, and decreased FTC133 and FTC238 cell proliferation (Fig. 1E and F).

Mounting evidence suggests that Klotho may be shed and could act as a circulating hormone, and it has been demonstrated that soluble Klotho and conditioned medium derived from Klotho-overexpressing cells are both active $(9,11,34,35)$. Therefore, FTC133 and FTC238 cells were next treated with $0.008 \mathrm{pmol} / 1$ soluble human KL1 (sKL; PeproTech Inc.). sKL was found to reduce the viability and proliferation of the FTC133 and FTC238 cells by 73 and 58\%, 69 and 56\%, respectively $(\mathrm{P}<0.05$, Fig. $1 \mathrm{E}$ and $\mathrm{F})$. The results showed that Klotho and sKL both significantly inhibited the survival and proliferation of the FTC133 and FTC238 cells.

Overexpression of Klotho increases the apoptosis of thyroid cancer cells. We next performed Nucleosome ELISA assay to assess the apoptosis of the FTC133 and FTC238 cells. The results revealed that both the Klotho-transfected and $\mathrm{sKL}$ treatment group exhibited more apoptotic cells when compared to that of the control group ( $\mathrm{P}<0.05$, Fig. $2 \mathrm{~A})$; no significant difference was noted between the pCDNA3.1 and control group ( $\mathrm{P}>0.05$, Fig. 2A). We next determined the activity of caspase- 3 in the different groups using the Caspase-Glo ${ }^{\circledR} 3 / 7$ assay. The activity of caspase- 3 was much higher in the Klotho-transfected and sKL treatment group when compared with that of the control group (Fig. 2B). The results indicated that Klotho and sKL both significantly promoted FTC133 and FTC238 cell apoptosis.

Loss of Klotho increases human cancer cell growth and inhibits cell apoptosis. We next investigated the effects of Klotho knockdown on the growth and apoptosis of thyroid cancer cells. To decrease the expression of Klotho protein, thyroid cancer cells were transfected with Klotho siRNA (siKL) or control siRNA (siMock). RT-PCR and western blot analysis results showed that Klotho expression in the thyroid cancer FTC133 and FTC238 cells was markedly decreased following siKL transfection $(\mathrm{P}<0.05$, Fig. $3 \mathrm{~A}$ and $\mathrm{B})$, whereas there was no significant difference between the siMock group and control group ( $\mathrm{P}>0.05)$. Moreover, cancer cell proliferation was markedly increased in the siKL-transfected cells compared with the cell proliferation noted in the control and siMock groups $(\mathrm{P}<0.05$, Fig. 3C). Simultaneously, cell apoptosis was also measured. The results showed that siKL transfection significantly inhibited the apoptosis of the FTC133 and FTC238 cells when compared to the apoptosis noted in the control group, whereas siMock transfection had no obvious effect on cell apoptosis (Fig. 3D). 
A

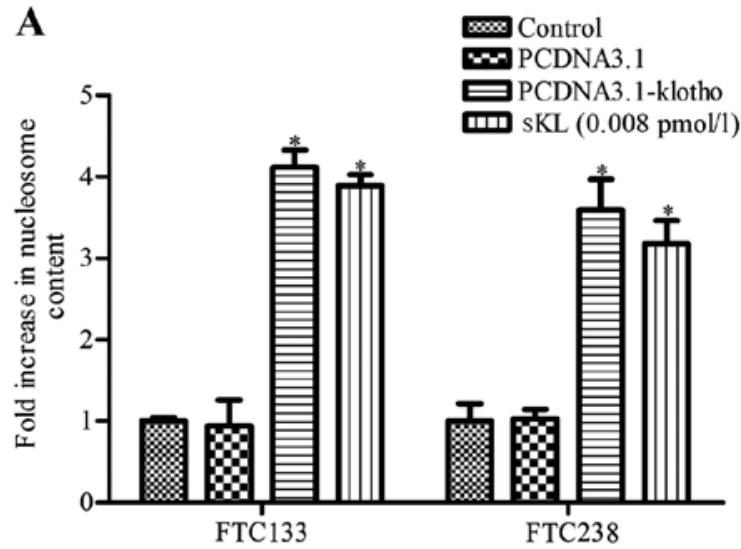

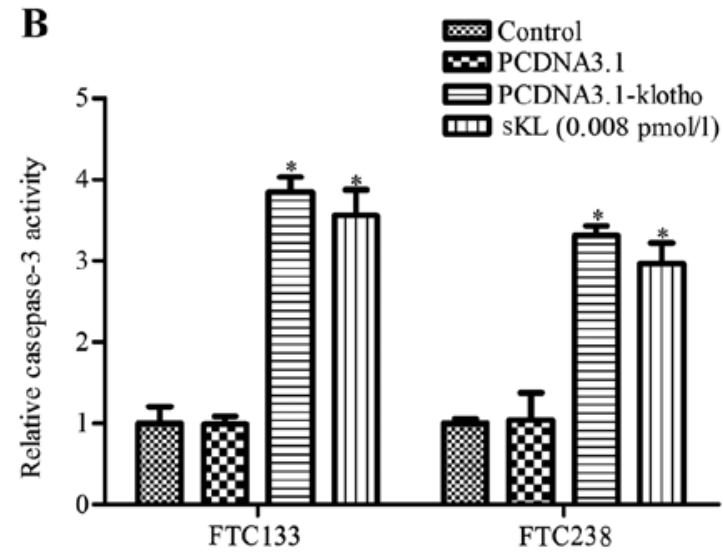

Figure 2. Effects of klotho overexpression on the apoptosis of thyroid cancer cells. (A) Cell apoptosis was assessed using the Nucleosome ELISA kit. (B) Caspase-Glo 3/7 assay was used to assess the activity of caspase-3. Cells were transfected with pCDNA3.1-Klotho, control vector pCDNA3.1 or treated with $0.008 \mathrm{pmol} / 1 \mathrm{sKL}$. Experiments were performed in triplicate, and data shown are the mean results $\pm \mathrm{SD} ;{ }^{*} \mathrm{P}<0.05$ compared with the control group.

A
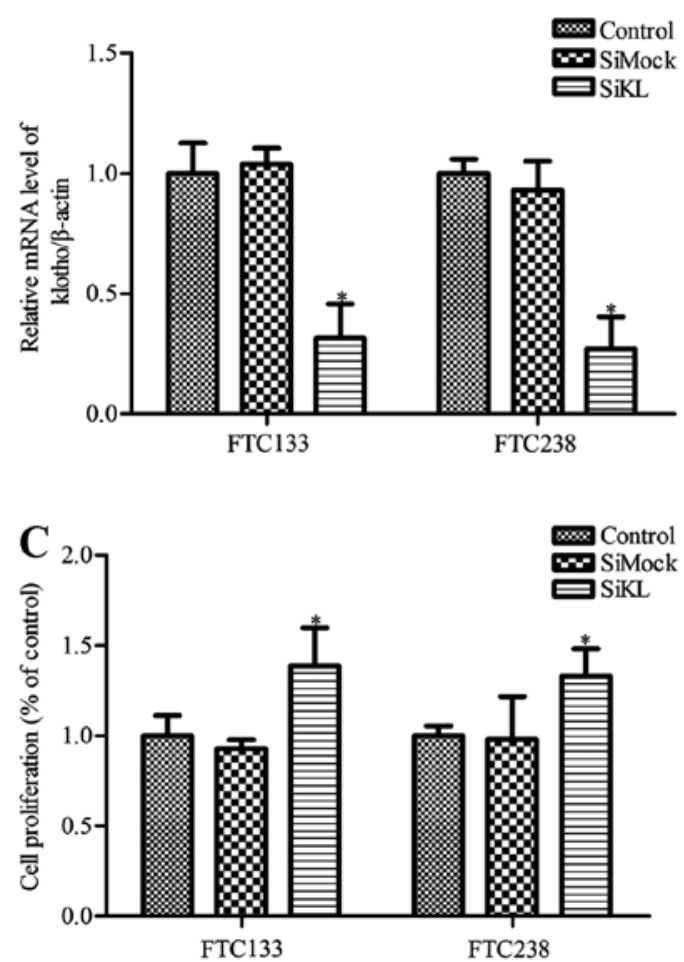

B
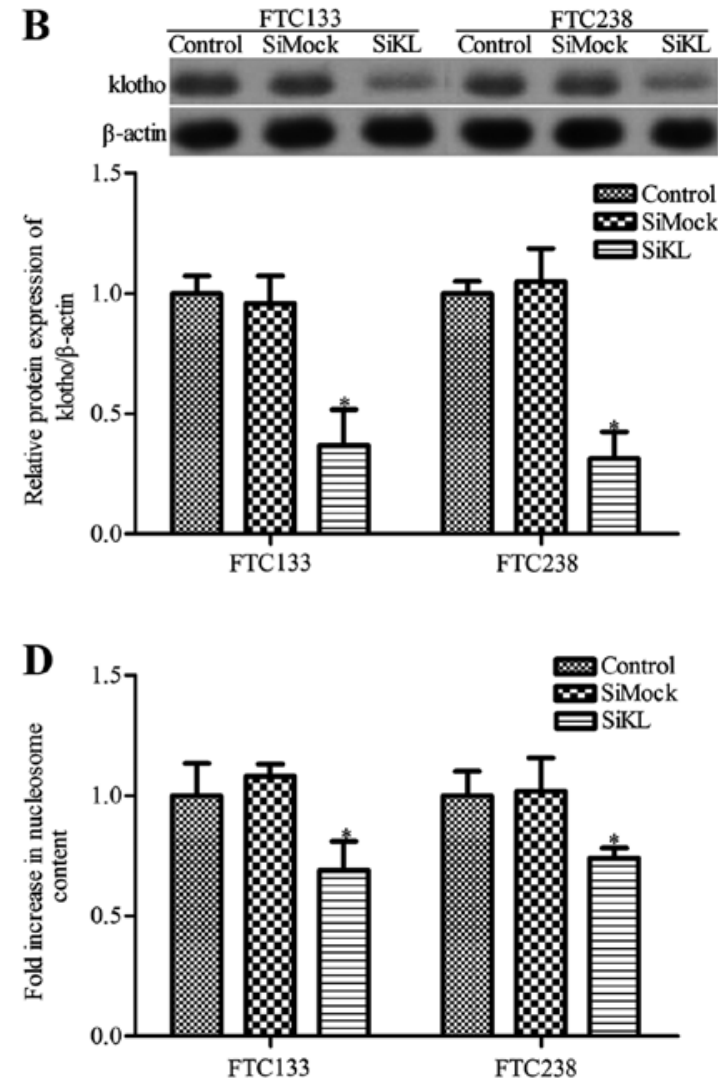

Figure 3. Loss of Klotho increases the proliferation and inhibits cell apoptosis in the FTC133 and FTC238 cells. (A) Klotho mRNA level was determined by RT-PCR. (B) Klotho protein expression was determined by western blot analysis. (C) Cell proliferation was assessed by the CCK-8 assay. (D) Cell apoptosis was carried out using the Nucleosome ELISA kit. Cells were transfected with siKL or siMock. Experiments were performed in triplicate, and data shown are the mean results $\pm \mathrm{SD} ;{ }^{*} \mathrm{P}<0.05$ compared with the control group.

Klotho inhibits STC1 expression in thyroid cancer cells. STC1 is a secreted glycoprotein hormone and a molecular marker for micrometastases of various human cancers, including thyroid cancer (27). Yahata et al showed that the Klotho gene regulates the expression of STC1 and STC2 in the kidney (19). To further detect the mechanism of Klotho-induced thyroid cancer cell apoptosis and growth inhibition, the effects of Klotho on the expression of STC1 and STC2 in thyroid cancer cell lines FTC133 and FTC238 were analyzed. RT-PCR and western blot analysis showed that STC1 mRNA and protein expression levels were significantly decreased in the Klothooverexpressing and sKL-treated thyroid cancer cell lines compared with the control group ( $\mathrm{P}<0.05$, Fig $4 \mathrm{~A}$ and $\mathrm{B})$. There was no obvious difference between the control group and control pcDNA3.1 vector group ( $\mathrm{P}>0.05$, Fig. $4 \mathrm{~A}$ and $\mathrm{B}$ ). Since STC1 is a secreted glycoprotein hormone, we also 


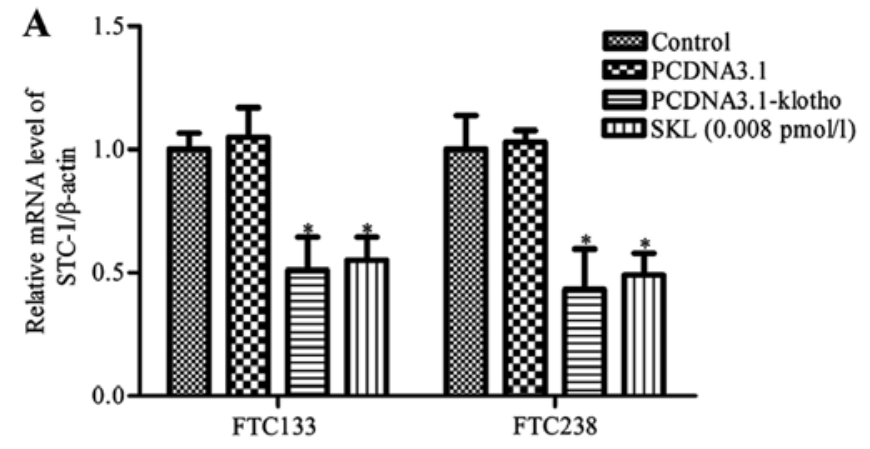

B
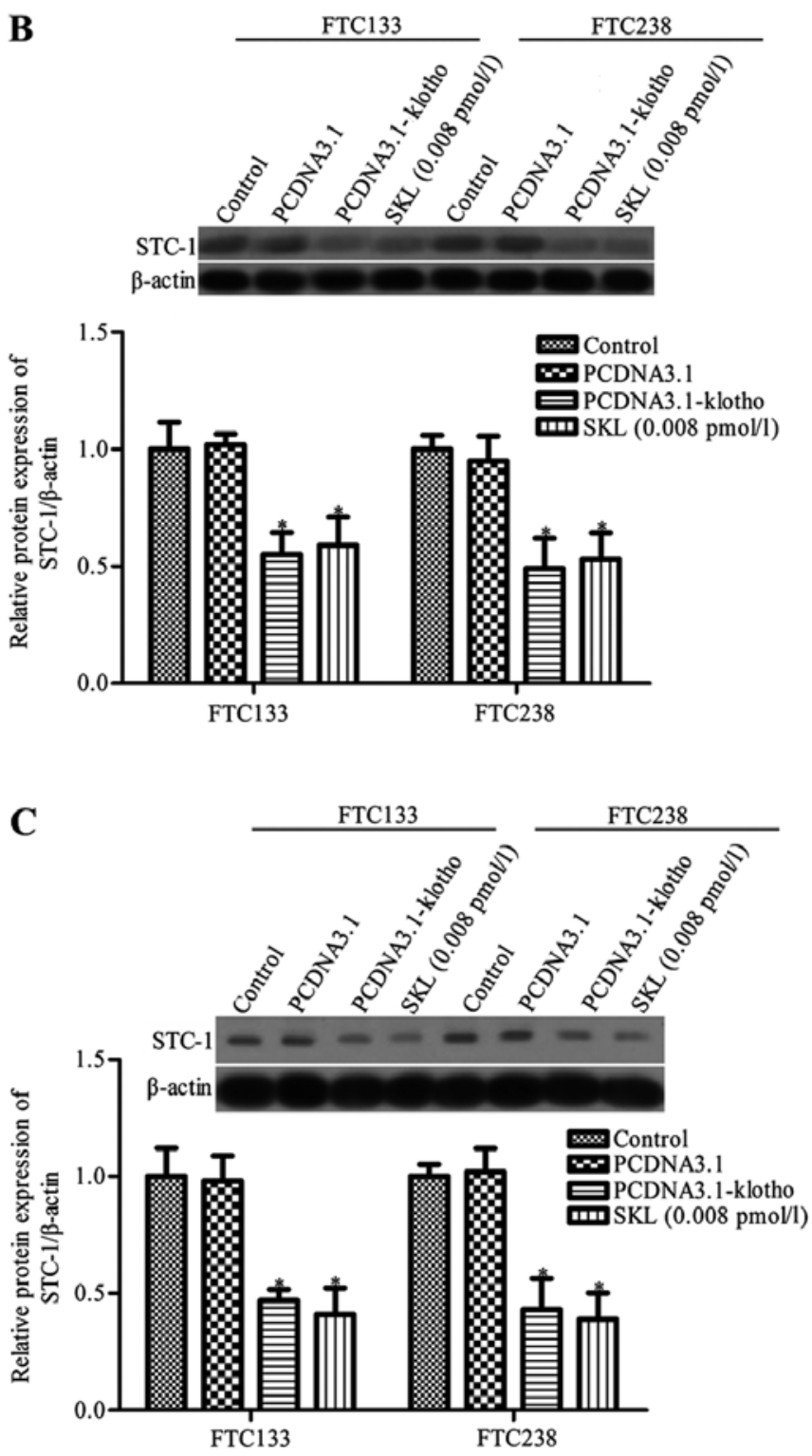

Figure 4. Effects of klotho overexpression on STC1 expression in thyroid cancer cells. (A and B) RT-PCR and western blot analysis were used to determine the mRNA (A) and protein level (B) of STC1 in the FTC133 and FTC238 cells. (C) The expression of STC1 in cell culture medium. Cells were transfected with pCDNA3.1-Klotho, control vector pCDNA3.1 or treated with $0.008 \mathrm{pmol} / 1 \mathrm{sKL}$. Experiments were performed in triplicate, and data shown are the mean results $\pm \mathrm{SD} ;{ }^{*} \mathrm{P}<0.05$ compared with the control group.

determined the protein expression of STC1 in cell culture medium. The results revealed that the secretion of STC1 in the Klotho-overexpressing and sKL-treated thyroid cancer cell lines was much lower than the secretion in the control group ( $\mathrm{P}<0.05$, Fig. 4C).

STC1 silencing inhibits cell growth and promotes cell apoptosis. Thyroid cancer cells were transfected with STC1 siRNA (siSTC1) or control siRNA (siMock), and the level of STC1 in the FTC133 and FTC238 cells was determined by RT-PCR and western blot analysis, respectively. As shown in Fig. 5A and B, STC1 mRNA and protein expression was significantly decreased in the siSTC1-treated thyroid cancer cells compared with that noted in the control and siMock groups $(\mathrm{P}<0.05)$. We also observed that STC1 silencing markedly inhibited FTC133 and FTC238 cell proliferation and induced cell apoptosis (Fig. 5C and D). To further ascertain whether STC1 is involved in Klotho-induced cell apoptosis, thyroid cancer cells were treated with $5 \mu \mathrm{l}$ recombinant human STC1 (hSTC1, $0.5 \mu \mathrm{g} / \mu \mathrm{l}$; BioVender Research and Diagnostic Products, Czech Republic). The results showed that hSTC1 treatment significantly promoted FTC133 and FTC238 cell proliferation and inhibited apoptosis. Moreover, hSTC1 treatment attenuated Klotho-induced inhibiiton of cell proliferation and promotion of apoptosis (Fig. 5C and D). These results indicate that Klotho inhibits cancer cell growth and induces apoptosis which is partially dependent on the downregulation of STC1 expression.

\section{Discussion}

It has been reported that STC1 is highly expression in several human tumors, including colorectal, breast, hepatocellular carcinomas and thyroid cancers (26-29). Klotho is known to regulate the expression of STC1. Mice deficient in Klotho expression exhibit increased renal gene expression of STC1 and STC2 compared with wild-type mice (19). Moreover, the level of Klotho was found to be downregulated in breast, lung, colon, pancreatic, gastric and cervical cancer (36-38). Although evidence suggests that Klotho acts as a tumor suppressor in numerous cancers (39), little is known concerning its potential effect on thyroid cancer.

We reported here that overexpression of Klotho in the thyroid cancer cell lines FTC133 and FTC238 resulted in a decreased capacity for cell proliferation and an increased capacity for cell apoptosis. To further demonstrate the effects of Klotho overexpression in FTC133 and FTC238 cells, caspase- 3 activity was determined using the Caspase-Glo 3/7 assay. Klotho may be shed and acts as a circulating hormone, and it has been demonstrated that soluble Klotho and conditioned medium derived from Klotho-overexpressing cells are both active $(9,11,34,35)$. We also found that treatment with $0.008 \mathrm{pmol} / 1 \mathrm{sKL}$ significantly reduced cell growth and promoted cell apoptosis. Loss of Klotho resulted in the promotion of thyroid cancer cell proliferation and inhibition of apoptosis. These results indicate that Klotho plays an important role in thyroid cancer.

Yahata et al showed that the Klotho gene regulates the expression of STC1 and STC2 in the kidney (19). Moreover, STC1 is upregulated in many cancers, including thyroid cancer. STC1 is an endocrine hormone firstly discovered from bony fishes, and is also expressed in mammals (40). There is mounting evidence that the mammalian STC1 gene is widely expressed in many tissues at different levels (mRNA 
A
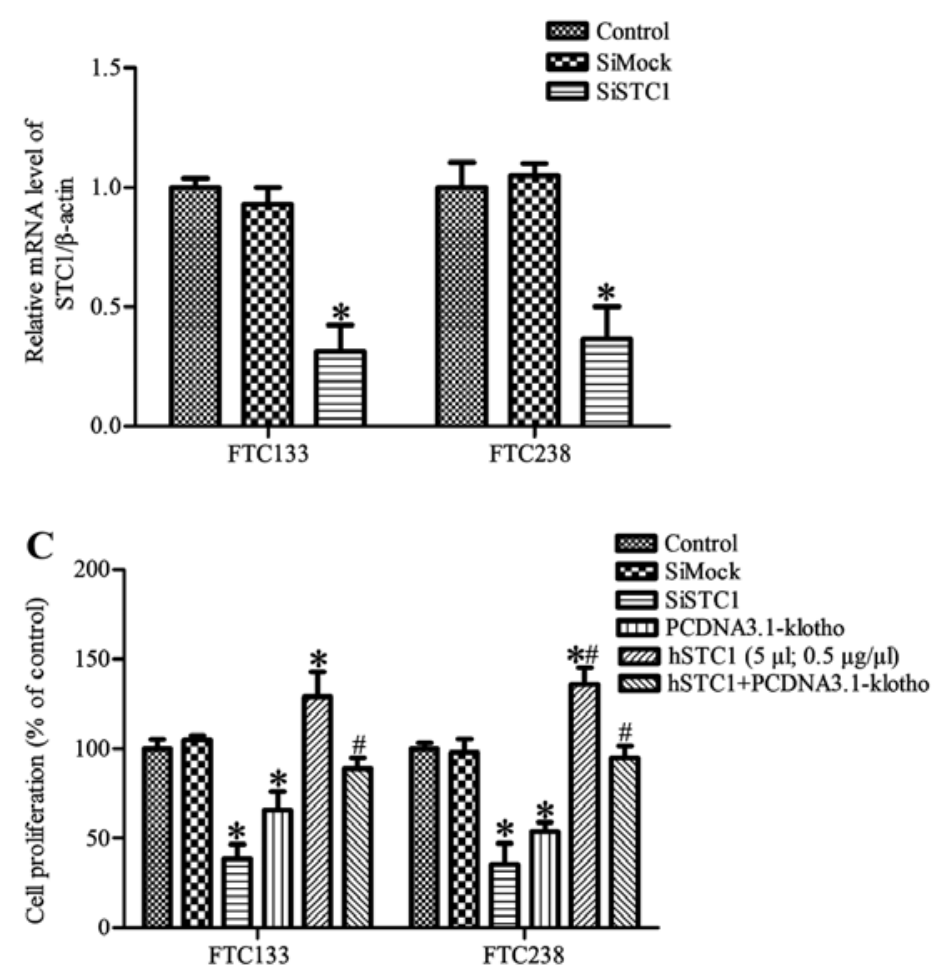

B
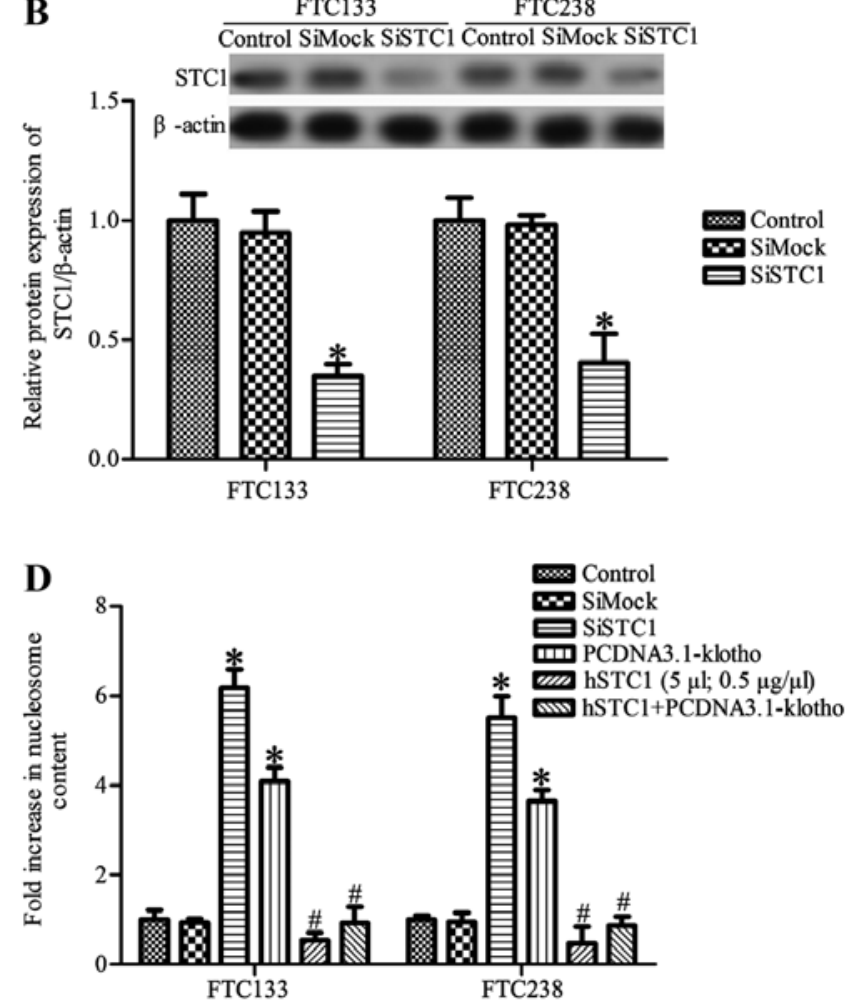

Figure 5. STC1 silencing inhibits cell growth and promotes cell apoptosis. (A) The STC1 mRNA level was determined by RT-PCR. (B) STC1 protein expression was determined by western blot analysis. (C) Cell proliferation was determined by the CCK-8 assay. (D) Cell apoptosis was carried out using the Nucleosome ELISA kit. Cells were transfected with siSTC1, siMock or treated with $2.5 \mu \mathrm{g}$ recombinant human STC1 (hSTC1). Experiments were performed in triplicate, data shown are the mean results $\pm \mathrm{SD} ;{ }^{*} \mathrm{P}<0.05$ compared with the control group, ${ }^{\prime} \mathrm{P}<0.05$ compared with the pCDNA3.1-klotho group.

or protein), and may play a different function. Moreover, evidence suggests that altered patterns of STC1 expression are tightly involved in the development of cancer (26). The present study showed that Klotho overexpression markedly inhibited the expression of STC1 in thyroid cancer FTC133 and FTC238 cells, which corroborated the findings reported in the literature. In addition, FTC133 and FTC238 cells transfected with siKL showed a marked increase in the expression of STC1. We next established STC1-silenced cells by STC1 siRNA. The results showed that STC1 knockdown induced the inhibition of proliferation and the promotion of apoptosis in the FTC133 and FTC238 cells. These results suggest that the effects of Klotho on FTC133 and FTC238 cell growth are dependent on the level of STC1. STC1 is a secreted glycoprotein hormone, and we observed that hSTC1 significantly inhibited Klotho overexpression-induced cell apoptosis. These data suggest that Klotho inhibits thyroid cancer cell proliferation and induces cell apoptosis dependent on STC1.

The present study also had several limitations. The detailed molecular mechanism of Klotho-mediated cell proliferation and apoptosis remains unclear. Furthermore, Klotho-induced cell apoptosis was only tested in thyroid cancer cell lines FTC133 and FTC238; it will require further validation in other types of thyroid cancer cell lines and in animal models. In summary, our study provides initial evidence that Klotho inhibits human follicular thyroid cancer cell growth and promotes apoptosis through regulation of the expression of STC1, and may be a new target for thyroid cancer treatment.

\section{Acknowledgements}

This study was supported by grants from the National Natural Science Foundation of China (81302003), the National Science and Technology Major Project (No. 2013ZX09303001) and the Science \& Technology Development Fund of Tianjin Education Commission for Higher Education (20120109).

\section{References}

1. Jemal A, Bray F, Center MM, Ferlay J, Ward E and Forman D: Global cancer statistics. CA Cancer J Clin 61: 69-90, 2011.

2. Xing M: Molecular pathogenesis and mechanisms of thyroid cancer. Nat Rev Cancer 13: 184-199, 2013.

3. Mazzaferri EL and Jhiang SM: Long-term impact of initial surgical and medical therapy on papillary and follicular thyroid cancer. Am J Med 97: 418-428, 1994.

4. Cornett WR, Sharma AK, Day TA, Richardson MS, Hoda RS, van Heerden JA and Fernandes JK: Anaplastic thyroid carcinoma: An overview. Curr Oncol Rep 9: 152-158, 2007.

5. Ain KB, Egorin MJ and DeSimone PA; Collaborative Anaplastic Thyroid Cancer Health Intervention Trials (CATCHIT) Group: Treatment of anaplastic thyroid carcinoma with paclitaxel: Phase 2 trial using ninety-six-hour infusion. Thyroid 10: 587-594, 2000.

6. Chen B, Wang X, Zhao W and Wu J: Klotho inhibits growth and promotes apoptosis in human lung cancer cell line A549. J Exp Clin Cancer Res 29: 99-105, 2010.

7. Chen L, Liu H, Liu J, Zhu Y, Xu L, He H, Zhang H, Wang S, Wu Q, Liu W, et al: Klotho endows hepatoma cells with resistance to anoikis via VEGFR2/PAK1 activation in hepatocellular carcinoma. PLoS One 8: e58413, 2013.

8. Shiraki-Iida T, Aizawa H, Matsumura Y, Sekine S, Iida A, Anazawa H, Nagai R, Kuro-o M and Nabeshima Y: Structure of the mouse klotho gene and its two transcripts encoding membrane and secreted protein. FEBS Lett 424: 6-10, 1998. 
9. Wolf I, Levanon-Cohen S, Bose S, Ligumsky H, Sredni B Kanety H, Kuro-o M, Karlan B, Kaufman B, Koeffler HP, et al: Klotho: A tumor suppressor and a modulator of the IGF-1 and FGF pathways in human breast cancer. Oncogene 27: 7094-7105, 2008.

10. Kuro-o M, Matsumura Y, Aizawa H, Kawaguchi H, Suga T, Utsugi T, Ohyama Y, Kurabayashi M, Kaname T, Kume E, et al: Mutation of the mouse klotho gene leads to a syndrome resembling ageing. Nature 390: 45-51, 1997.

11. Matsumura Y, Aizawa H, Shiraki-Iida T, Nagai R, Kuro-o M and Nabeshima Y: Identification of the human klotho gene and its two transcripts encoding membrane and secreted klotho protein Biochem Biophys Res Commun 242: 626-630, 1998.

12. Nagai T, Yamada K, Kim HC, Kim YS, Noda Y, Imura A Nabeshima $Y$ and Nabeshima T: Cognition impairment in the genetic model of aging klotho gene mutant mice: A role of oxidative stress. FASEB J 17: 50-52, 2003.

13. Nagai R, Saito Y, Ohyama Y, Aizawa H, Suga T, Nakamura T, Kurabayashi $M$ and Kuroo M: Endothelial dysfunction in the klotho mouse and downregulation of klotho gene expression in various animal models of vascular and metabolic diseases. Cell Mol Life Sci 57: 738-746, 2000.

14. Saito Y, Yamagishi T, Nakamura T, Ohyama Y, Aizawa $\mathrm{H}$, Suga T, Matsumura Y, Masuda H, Kurabayashi M, Kuro-o M, et al: Klotho protein protects against endothelial dysfunction. Biochem Biophys Res Commun 248: 324-329, 1998.

15. Arking DE, Krebsova A, Macek M Sr, Macek M Jr, Arking A, Mian IS, Fried L, Hamosh A, Dey S, McIntosh I, et al: Association of human aging with a functional variant of klotho. Proc Natl Acad Sci USA 99: 856-861, 2002.

16. Tao Y, Pinzi V, Bourhis J and Deutsch E: Mechanisms of disease: Signaling of the insulin-like growth factor 1 receptor pathway - therapeutic perspectives in cancer. Nat Clin Pract Oncol 4: 591-602, 2007.

17. Mattarocci S, Abbruzzese C, Mileo AM, Visca P, Antoniani B, Alessandrini G, Facciolo F, Felsani A, Radulescu RT and Paggi MG: Intracellular presence of insulin and its phosphorylated receptor in non-small cell lung cancer. J Cell Physiol 221: 766-770, 2009

18. Chang B, Kim J, Jeong D, Jeong Y, Jeon S, Jung SI, Yang Y, Kim KI, Lim JS, Kim C, et al: Klotho inhibits the capacity of cell migration and invasion in cervical cancer. Oncol Rep 28 : 1022-1028, 2012

19. Yahata K, Mori K, Mukoyama M, Sugawara A, Suganami T, Makino H, Nagae T, Fujinaga Y, Nabeshima Y and Nakao K: Regulation of stanniocalcin 1 and 2 expression in the kidney by klotho gene. Biochem Biophys Res Commun 310: 128-134, 2003

20. Chang AC, Jeffrey KJ, Tokutake Y, Shimamoto A, Neumann AA, Dunham MA, Cha J, Sugawara M, Furuichi Y and Reddel RR Human stanniocalcin (STC): Genomic structure, chromosomal localization, and the presence of CAG trinucleotide repeats. Genomics 47: 393-398, 1998.

21. Wagner GF, Hampong M, Park CM and Copp DH: Purification, characterization, and bioassay of teleocalcin, a glycoprotein from salmon corpuscles of Stannius. Gen Comp Endocrinol 63 481-491, 1986.

22. Hayase S, Sasaki Y, Matsubara T, Seo D, Miyakoshi M, Murata T, Ozaki T, Kakudo K, Kumamoto K, Ylaya K, et al: Expression of stanniocalcin 1 in thyroid side population cells and thyroid cancer cells. Thyroid 25: 425-436, 2015.

23. Yeung BH, Law AY and Wong CK: Evolution and roles of stanniocalcin. Mol Cell Endocrinol 349: 272-280, 2012.

24. Varghese R, Wong CK, Deol H, Wagner GF and DiMattia GE: Comparative analysis of mammalian stanniocalcin genes. Endocrinology 139: 4714-4725, 1998 .
25. Chang AC, Janosi J, Hulsbeek M, de Jong D, Jeffrey KJ, Noble JR and Reddel RR: A novel human cDNA highly homologous to the fish hormone stanniocalcin. Mol Cell Endocrinol 112: 241-247, 1995.

26. Chang AC, Jellinek DA and Reddel RR: Mammalian stanniocalcins and cancer. Endocr Relat Cancer 10: 359-373, 2003.

27. Fujiwara Y, Sugita Y, Nakamori S, Miyamoto A, Shiozaki K, Nagano H, Sakon M and Monden M: Assessment of stanniocalcin-1 mRNA as a molecular marker for micrometastases of various human cancers. Int J Oncol 16: 799-804, 2000.

28. McCudden CR, Majewski A, Chakrabarti S and Wagner GF: Co-localization of stanniocalcin-1 ligand and receptor in human breast carcinomas. Mol Cell Endocrinol 213: 167-172, 2004

29. Okabe H, Satoh S, Kato T, Kitahara O, Yanagawa R, Yamaoka Y, Tsunoda T, Furukawa Y and Nakamura Y: Genome-wide analysis of gene expression in human hepatocellular carcinomas using cDNA microarray: Identification of genes involved in viral carcinogenesis and tumor progression. Cancer Res 61: 2129-2137, 2001.

30. Chen B, Ma X, Liu S, Zhao W and Wu J: Inhibition of lung cancer cells growth, motility and induction of apoptosis by Klotho, a novel secreted Wnt antagonist, in a dose-dependent manner. Cancer Biol Ther 13: 1221-1228, 2012.

31. Shih A, Davis FB, Lin HY and Davis PJ: Resveratrol induces apoptosis in thyroid cancer cell lines via a MAPK- and p53-dependent mechanism. J Clin Endocrinol Metab 87: 1223-1232, 2002

32. Livak KJ and Schmittgen TD: Analysis of relative gene expression data using real-time quantitative PCR and the $2^{-\Delta \Delta C T}$ method. Methods 25: 402-408, 2001.

33. Liu G, Yang G, Chang B, Mercado-Uribe I, Huang M, Zheng J, Bast RC, Lin SH and Liu J: Stanniocalcin 1 and ovarian tumorigenesis. J Natl Cancer Inst 102: 812-827, 2010.

34. Abramovitz L, Rubinek T, Ligumsky H, Bose S, Barshack I, Avivi C, Kaufman B and Wolf I: KL1 internal repeat mediates klotho tumor suppressor activities and inhibits bFGF and IGF-I signaling in pancreatic cancer. Clin Cancer Res 17: 4254-4266, 2011.

35. Chen CD, Podvin S, Gillespie E, Leeman SE and Abraham CR: Insulin stimulates the cleavage and release of the extracellular domain of Klotho by ADAM10 and ADAM17. Proc Natl Acad Sci USA 104: 19796-19801, 2007.

36. Lee J, Jeong DJ, Kim J, Lee S, Park JH, Chang B, Jung SI, Yi L, Han Y, Yang Y, et al: The anti-aging gene KLOTHO is a novel target for epigenetic silencing in human cervical carcinoma. Mol Cancer 9: 109, 2010.

37. Wang L, Wang X, Wang X, Jie P, Lu H, Zhang S, Lin X, Lam EK, Cui Y, Yu J, et al: Klotho is silenced through promoter hypermethylation in gastric cancer. Am J Cancer Res 1: 111-119, 2011.

38. Pan J, Zhong J, Gan LH, Chen SJ, Jin HC, Wang X and Wang LJ Klotho, an anti-senescence related gene, is frequently inactivated through promoter hypermethylation in colorectal cancer. Tumour Biol 32: 729-735, 2011.

39. Liu H, Fergusson MM, Castilho RM, Liu J, Cao L, Chen J, Malide D, Rovira II, Schimel D, Kuo CJ, et al: Augmented Wnt signaling in a mammalian model of accelerated aging. Science 317: 803-806, 2007.

40. Yeung HY, Lai KP, Chan HY, Mak NK, Wagner GF and Wong CK: Hypoxia-inducible factor-1-mediated activation of stanniocalcin-1 in human cancer cells. Endocrinology 146: 4951-4960, 2005. 